Aim of the study: To evaluate the prognostic role of markers of fluor18-fluorodeoxyglucose positron emission tomography with computed tomography $\left({ }^{18} \mathrm{~F}-\mathrm{FDG}-\mathrm{PET}-\mathrm{CT}\right)$, such as maximum standard uptake value $\left(S U V_{\text {max }}\right)$ and metabolic tumour volume (MTV) measured at primary and nodal disease, and their clinical significance in terms of predicting treatment outcomes and survival.

Material and methods: Between January 2017 and January 2020, 20 case records of nasopharyngeal carcinoma patients who underwent ${ }^{18} \mathrm{~F}$-FDG-PET-CT as part of staging workup before radiotherapy and as a part of response evaluation after radiotherapy were retrospectively reviewed.

Results: At a median follow-up of 34.7 months, the 2-year progression-free survival (PFS) was $70 \%$ and 2-year overall survival (OS) was $79 \%$. Patients with a lower nodal SUV $\left(S_{\text {SUa }-\mathrm{N}}\right)$ had a better 2-year PFS $(91 \%$ vs. $46 \% ; p=0.035)$ and 2 -year OS $(95 \%$ vs. $58 \% ; p=0.015)$. A high SUV of $>10.58$ was a negative predictor of OS (95\% confidence interval [Cl]: $0.93-1 ; p=0.003)$ as well as PFS $(95 \%$ Cl: $0.64-1 ; p=0.017)$. Also, a high MTV $>25.8 \mathrm{~cm}^{3}$ was a negative predictor of PFS (95\% Cl: 0.58-0.98; $p=0.048$ ). MTV was an independent predictor of PFS and OS on univariate analysis, whereas it was not significant in the Cox regression multivariate analysis. Conclusions: High values of MTV and SUV $_{\text {max-N }}$ can be considered as independent prognostic factors of OS and PFS in nasopharyngeal cancer patients treated with concurrent chemoradiation, highlighting the need for more intensified treatment.

Key words: nasopharyngeal carcinoma, SUV ${ }_{\max }$, MTV, prognostic factor.

Contemp Oncol (Pozn) 2021; 25 (3): 153-159 DOI: https://doi.org/10.5114/wo.2021.109620

\section{Prognostic significance of metabolic tumour volume and maximum standard uptake value of fluor-18-fluorodeoxyglucose positron emission tomography with computed tomography in nasopharyngeal carcinoma}

Christopher John ${ }^{1}$, Jeyaanth Venkatasai ${ }^{1}$, Satish Srinivas Kondaveeti ${ }^{1}$, Arunan Murali², Gokulakrishnan Periakaruppan ${ }^{2}$, Venkatachalapathy $\mathrm{E}^{3}$, Manickavasagam Meenakshisundaram ${ }^{4}$, Ravi Chandran Ambalathandi ${ }^{4}$, Hemavathi Masilamani ${ }^{1}$

${ }^{1}$ Department of Radiation Oncology, Sri Ramachandra Institute of Higher Education and Research, Porur, Chennai, India

${ }^{2}$ Department of Radio-Diagnosis, Sri Ramachandra Institute of Higher Education and Research, Porur, Chennai, India

${ }^{3}$ Department of Nuclear Medicine, Sri Ramachandra Institute of Higher Education and Research, Porur, Chennai, India

${ }^{4}$ Department of Medical Oncology, Sri Ramachandra Institute of Higher Education and Research, Porur, Chennai, India

\section{Introduction}

The standard of care in nasopharyngeal carcinoma (NPC) management is concurrent chemoradiation followed by adjuvant chemotherapy. Nasopharyngeal carcinoma differs from other head and neck cancers by virtue of its unique clinical behaviour and aetiological differences. This malignancy has shown higher incidence in Southeast Asia (annual incidence rate [AIR] of 6.4/100,000 in males and 2.4/100,000 in females). In India, its geographic distribution is heterogeneous, with higher incidence in Northeastern states (AIR of 19.4/100,000 population) [1]. Data from our hospital registry from South India revealed a lower prevalence of this condition in comparison to other head and neck malignancies

Currently, as per World Health Organisation pathological classification, these malignancies are categorized into keratinising squamous cell carcinoma and non-keratinising carcinoma variants [2]. However, there is no clear demonstration of clinical improvement when a specific histological subtype is considered as a prognostic predictor. Although outcomes in terms of local control have significantly improved using treatment protocols like chemo radiation as well as newer radiotherapy treatment techniques such as intensity-modulated radiotherapy (IMRT) [3], distant failure rates continue to be dismal in the locally advanced subset [4, 5]. Hence, various other prognostic factors such as Ebstein-Barr virus-related biomarkers are being hypothesised and evaluated $[6,7]$. However, there is still a growing need to analyse other indices to quantify prognosis.

Fluor-18-fluorodeoxyglucose positron emission tomography with computed tomography ( ${ }^{18} \mathrm{~F}-\mathrm{FDG}-\mathrm{PET}-\mathrm{CT}$ ) has been used as the preferred imaging modality for staging nasopharyngeal cancer patients [8]. Apart from its use as a staging modality, the use of the same as a prognostic marker has been explored in a few studies $[9,10]$. The standard uptake value maximum 
$\left(S U V_{\max }\right)$ for primary tumour and nodal disease has been explored as a potential prognostic biomarker for nasopharyngeal cancers; however, a uniform consensus on the same is still lacking $[11,12]$. The drawback of SUV $V_{\max }$ is that it represents the areas of active uptake within the tumour but does not provide an estimate of the entire tumour volume; hence, other metabolic parameters such as metabolic tumour volume (MTV) are also being studied to overcome the above-mentioned limitations $[13,14]$.

In this study, we have calculated the value of MTV, and SUV max $_{\text {of }}$ primary tumour (SUV max. $_{\text {P }}$ ) and nodal disease $\left(S U V_{\max -N}\right)$ in pre-treatment PET-CT of patients with non-metastatic nasopharyngeal cancer treated with chemoradiation and correlation were drawn in terms of treatment response, overall survival (OS) and progression-free survival (PFS). Thereby we evaluated the role of these PET-CT metabolic parameters as a prognostic tool in non-metastatic nasopharyngeal cancer.

\section{Material and methods}

Patient population: We retrospectively analysed image datasets and follow-up data of NPC patients who presented at our institute between January 2017 and January 2020. Informed consent was obtained from each patient prior to radiotherapy and chemotherapy on the use of their data and image data for subsequent research.

Eligible patients were those with biopsy-proven nasopharyngeal carcinoma, including those who had received concurrent chemoradiation with IMRT followed by adjuvant chemotherapy. Pre-therapy evaluation included those who had been evaluated with ${ }^{18}$ F-FDG-PET-CT as part of the staging and metastatic workup and had subsequently been staged in accordance with $8^{\text {th }}$ edition of the American Joint Committee on Cancer Staging Manual (2016). Patients with a Karnofsky index value below $70 \%$ and a prior history of other malignancies were excluded from the study.

${ }^{18}$ F-FDG-PET-CT parameters: the patients were administered an intravenous injection of ${ }^{18} \mathrm{~F}$-FDG-PET-CT at a dose of $0.22 \mathrm{mCi}(8.14 \mathrm{MBq}) / \mathrm{kg}(10-15 \mathrm{mCi} / 370-555 \mathrm{MBq})$ FDG. Forty-five minutes after the injection, patients were imaged from vertex to knee level with a $120 \mathrm{kV}, 50 \mathrm{~mA}$ CT scan with a slice thickness of $3 \mathrm{~mm}$ using a Siemens Biograph Horizon device.

Treatment details: For radiotherapy planning, the patients were simulated in the supine position and immobilised with a thermoplastic mould. A CT simulation was performed with a slice thickness of $3 \mathrm{~mm}$ extending from the vertex to the D8 spine using a Siemens Biograph Horizon. The contouring was done with PET fusion-based delineation as per the international guidelines by Lee et al. [15]. Radiotherapy was administered to a total dose of 66 Gy to the GTV, 60 Gy to the high-risk clinical target volumes, and to a dose of 54 Gy in elective nodal regions in 1.8-2 Gy daily fractionation. With regard to chemotherapy, the patients were administered cisplatin (CDDP) $40 \mathrm{mg} / \mathrm{m}^{2}$ weekly concurrent with radiation and adjuvant chemotherapy using CDDP $\left(70 \mathrm{mg} / \mathrm{m}^{2}\right)$ and 5FU (1000 mg/m² D1-5) 3-weekly following concurrent chemoradiation.
Follow-up: All patients were reviewed weekly during RT to assess the acute reactions, and post-therapy assessment with PET-CT was performed at 3 months postRT after completion of adjuvant chemotherapy. Response assessment was performed in accordance with PERCIST criteria.

${ }^{18}$ F-FDG-PET-CT Metabolic Parameters: The SUV values were obtained using attenuation-corrected images, patient body weight, amount of FDG injected, and cross-calibration factors between the dose calibrator and FDG-PET CT.

The MTV of the primary tumour was calculated and derived automatically under a fixed threshold of the SUV at 2.5. The contouring margin contained the entire primary MTV $_{2.5}$, where the MTV was a quantitative measurement of ${ }^{18} \mathrm{~F}$-FDG uptake within the tumour lesions and volume of interest.

Study design and statistical analysis: To evaluate the role of MTV and SUV ${ }_{\max }$ of the primary and node and its significance in terms of treatment outcomes: OS and PFS. The software used for statistical analysis was SPSS version 11, the survival fractions were estimated using the Kaplan-Meier method, and its correlation with treatment response and prognostic factors were analysed using the log-rank test. Statistical significance was set at $p<0.05$. Chi-square and Fisher's exact test were used to compare characteristics between the 2 groups SUV $V_{\text {max }-\mathrm{P}}$ and SUV $V_{\max -\mathrm{N}^{*}}$

\section{Results}

\section{Patient characteristics}

The patient characteristics are shown in Table 1, and a total of 20 patients were analysed in this study, of whom 17 were alive at the last follow-up. The median follow-up period was 34.7 months (range, 20-48 months). The common histological variant was non-keratinising carcinoma, comprising of $85 \%$, with most patients presenting in the locally advanced stages (III and IVA). The median nodal size measured on the short axis was $20 \mathrm{~mm}$ (range, 8-42 mm).

\section{Maximum standard uptake cut-off value}

Based on pre-therapy PET-CT, the mean SUV $V_{\text {max-P }}$ was 13.55, (range, 8.75 to 26.17 ) and the mean value of

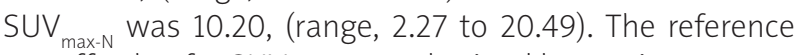
cut-off value for SUV $V_{\max -\mathrm{N}}$ was obtained by receiver operating characteristics (ROC) curve analysis for progression for a value of 10.58 (area under curve - AUC, $0.845 ; p=0.017$ ). In terms of MTV, the mean value was $22.04 \mathrm{~cm}^{3}$ (range, 8.53-56.92) and the reference cut-off value was $25.8 \mathrm{~cm}^{3}$ (AUC, $0.786 ; p=0.048$ ) by ROC curve analysis for progression as shown in Figure 1.

Based on the cut-off values for SUV max $_{\text {at }}$ at primary and node and MTV calculated using the ROC method, the distribution of these values with respect to disease stage and pathological variants in our study is depicted in Table 2.

\section{Prognostic significance of maximum standard uptake value and metabolic tumour volume treatment outcomes}

In terms of treatment outcomes, patients were evaluated with post-therapy PET-CT at 3 months post-radiotherapy. 
Among the 20 patients analysed, 14 (70\%) had complete metabolic response, 2 (10\%) were partial responders, and the remaining 4 (20\%) had progressive disease.

When stratified and compared against the average SUV $V_{\text {max-p }}$, there were no significant differences in response rates $(p=1.0)$. However, in terms of SUV $\mathrm{max}-\mathrm{N}$, there was a significant difference of $91.7 \%$ versus $37.5 \%$ in terms of complete response rates for patients with SUV $<10.58$, showingsignificantlybetterresponserates $(p=0.05)$. In terms of histological variants, the poorly differentiated variant had a poorer outcome, as shown in Table 3 (statistically insignificant). The MTV cut-off value of $25.8 \mathrm{~cm}^{3}$ was also statistically significant in terms of complete responders $(91.7 \%$ vs. $37.5 \% p=0.018)$

During the follow-up period, 2 patients who had partial response developed progressive disease ( 6 out of $n=20$, $30 \%)$ of whom 4 patients had succumbed to the disease, 1 patient had distant progression, and 1 had local progression, and they were salvaged with second-line chemotherapy. Among the above-mentioned patients who had progressed, 5 had an SUV max-N $_{\text {of }}>10.58$ and an MTV of $>25.8 \mathrm{~cm}^{3}$. The sensitivity and specificity regarding the use of these metabolic markers for prognostic significance is shown in Table 4.

\section{Survival outcomes}

The 2-year PFS and OS for all patients were 70\% and 79\%, respectively. In univariate analysis, high $S U V_{\text {max-p }}>13.55$ did not show any significant difference in terms of OS and PFS ( $p=0.582)$.

However, a higher SUV ${ }_{\text {max-N }}$ level > 10.58 was a negative prognostic factor. The 2-year PFS was (91\% vs. 46\%; $p=0.035)$ and the 2 -year OS was (95\% vs. $58 \% ; p=0.015$ ) for the SUV $\mathrm{max}-\mathrm{N}<10.58$ and SUV $\mathrm{Sax}_{\text {m N }}>10.58$, respectively, which was statistically significant (Table 5). When the $\mathrm{SUV}_{\text {max-N }}$ was stratified by stage or across histologies, there was no difference in PFS or OS $(p=0.715)$. A high SUV $V_{\max -\mathrm{N}}$ of > 10.58 was a negative predictor of OS ( $95 \%$ confidence

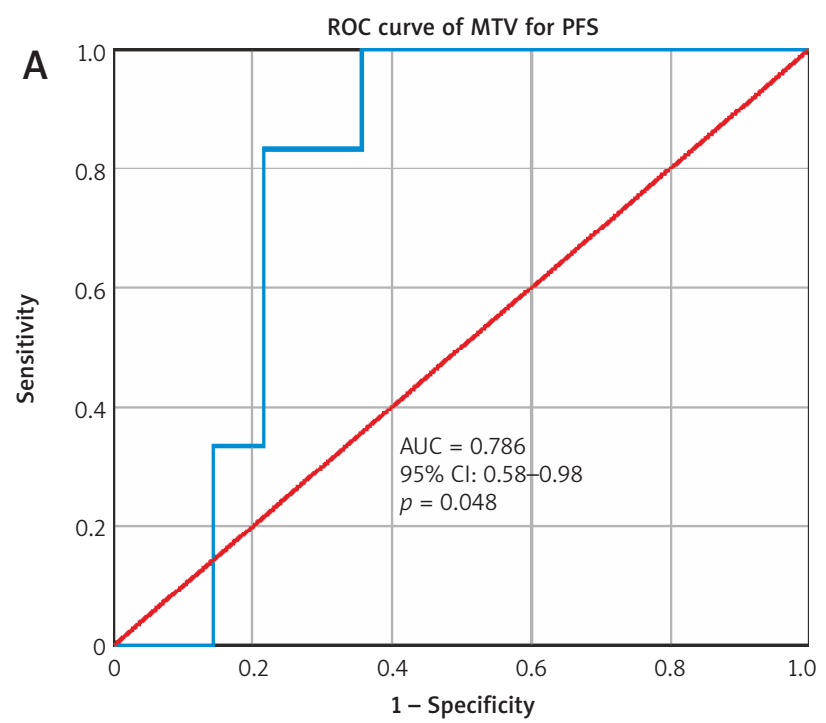

Table 1. Patient characteristics

\begin{tabular}{lc}
\hline Characteristics & $\begin{array}{c}\text { Number } \\
\text { of patients (\%) }\end{array}$ \\
Sex & $13(65)$ \\
Male & $7(35)$ \\
Female & \\
Age & $11(55)$ \\
$<45$ & $9(45)$ \\
$>45$ & \\
Pathology & $3(15)$ \\
SCC & $8(40)$ \\
Poorly differentiated non-keratinising carcinoma & $9(45)$ \\
Un-differentiated non-keratinising carcinoma & \\
T stage & $4(20)$ \\
T1-T2 & $16(80)$ \\
T3-T4 & \\
N stage & $7(35)$ \\
N0-N1 & $13(65)$ \\
N2-N3 & \\
Stage group & $2(10)$ \\
I-II & $18(90)$ \\
III-IVA & \\
Vaues are presented as number (\%) SCC - sauamous cel carcha
\end{tabular}

Values are presented as number (\%), SCC - squamous cell carcinoma

interval $[\mathrm{Cl}]$ : $0.93-1 ; p=0.003)$ as well as PFS $(95 \% \mathrm{Cl}$ : $0.64-1 ; p=0.017$ ), as shown in Figure 2.

In terms of MTV, the 2-year PFS was (90\% vs. 38\%; $p=0.006)$ and 2 -year OS was (99\% vs. 55\%; $p=0.008)$ for the cut-off values of MTV $\leq 25.8 \mathrm{~cm}^{3}$ and MTV $>25.8 \mathrm{~cm}^{3}$, respectively. A high MTV of $>25.8$ was also a negative predictor of OS ( $95 \% \mathrm{Cl}$ : $0.56-0.97 ; p=0.108)$ as well as PFS (95\% Cl: 0.58-0.98; $p=0.048$ ). The Kaplan-Meier curves for PFS and OS are shown in Figures 3 and 4.

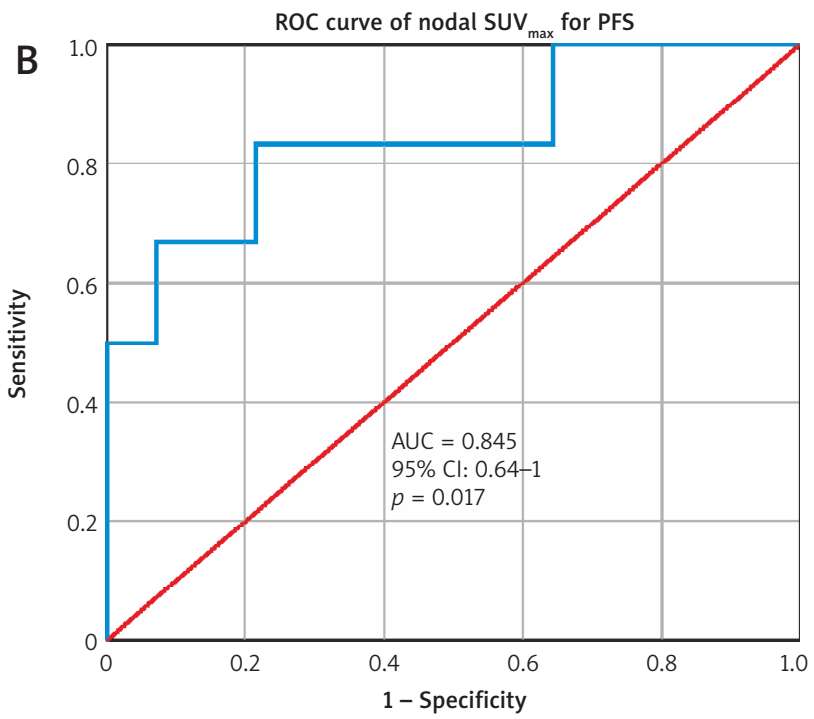

Fig. 1. Receiver operating characteristics curve for metabolic tumour volume and nodal maximum standard uptake value for predicting progression 
Table 2. Characteristics mapped to maximum standard uptake values

\begin{tabular}{|c|c|c|c|c|c|c|}
\hline \multirow[t]{3}{*}{ Characteristics } & \multicolumn{2}{|c|}{ SUV $_{\text {max-P }}$} & \multicolumn{2}{|c|}{$\mathrm{SUV}_{\max -\mathrm{N}}$} & \multicolumn{2}{|c|}{ MTV $\left(\mathrm{cm}^{3}\right)$} \\
\hline & $\leq 13.55$ & $>13.55$ & $\leq 10.58$ & $>10.58$ & $\leq 25.8$ & $>25.8$ \\
\hline & $n=10$ & $n=10$ & $n=12$ & $n=8$ & $n=12$ & $n=8$ \\
\hline \multicolumn{7}{|l|}{ Histology } \\
\hline SCC & $0(0 \%)$ & $4(40 \%)$ & $2(16.7 \%)$ & $2(25 \%)$ & $3(25 \%)$ & $1(12.5 \%)$ \\
\hline $\begin{array}{l}\text { Poorly differentiated } \\
\text { carcinoma }\end{array}$ & $6(60 \%)$ & $1(10 \%)$ & $3(25 \%)$ & $4(50 \%)$ & $4(33.3 \%)$ & $3(37.5 \%)$ \\
\hline $\begin{array}{l}\text { Un-differentiated non- } \\
\text { keratinising carcinoma }\end{array}$ & $4(40 \%)$ & $5(50 \%)$ & 7 (58.3\%) & $2(25 \%)$ & 5 (41.7\%) & $4(50 \%)$ \\
\hline \multicolumn{7}{|l|}{ MTV stage } \\
\hline $\mathrm{T} 1-\mathrm{T} 2$ & $2(20 \%)$ & $2(20 \%)$ & $4(33.3 \%)$ & $0(0 \%)$ & $3(25 \%)$ & $1(12.5 \%)$ \\
\hline T3-T4 & $8(80 \%)$ & $8(80 \%)$ & $8(66.7 \%)$ & $8(100 \%)$ & $9(75 \%)$ & 7 (87.5\%) \\
\hline NO-N1 & $3(30 \%)$ & $4(40 \%)$ & $6(50 \%)$ & $1(12.5 \%)$ & $5(41.7 \%)$ & $2(25 \%)$ \\
\hline N2vN3 & $7(70 \%)$ & $6(60 \%)$ & $6(50 \%)$ & 7 (8.75\%) & 7 (58.3\%) & $6(75 \%)$ \\
\hline \multicolumn{7}{|l|}{ Stage group } \\
\hline$|-| \mid$ & $0(0 \%)$ & $2(20 \%)$ & $2(16.7 \%)$ & $0(0 \%)$ & $1(8.3 \%)$ & $1(12.5 \%)$ \\
\hline III-IVA & $10(100 \%)$ & $8(80 \%)$ & $10(83.3 \%)$ & $8(100 \%)$ & 11 (91.7\%) & $7(87.5 \%)$ \\
\hline
\end{tabular}

Values are presented as number (\%), SUV - standard uptake value, MTV - metabolic tumour volume

Table 3. Treatment outcomes

\begin{tabular}{|c|c|c|c|c|c|}
\hline \multirow[t]{2}{*}{ Prognostic parameters } & \multirow[t]{2}{*}{ No of patients } & \multicolumn{3}{|c|}{ Treatment response } & \multirow[t]{2}{*}{$p$-value } \\
\hline & & CR (\%) & PR (\%) & PD (\%) & \\
\hline Primary & & & & & 1.00 \\
\hline SUV $V_{\text {max }-\mathrm{P}} \leq 13.55$ & 10 & $7(70)$ & $1(10)$ & $2(20)$ & \\
\hline SUV $_{\text {max }-\mathrm{P}}>13.55$ & 10 & $7(70)$ & $1(10)$ & $2(20)$ & \\
\hline NODE & & & & & 0.05 \\
\hline $\mathrm{SUV}_{\max -\mathrm{N}} \leq 10.58$ & 12 & $11(91.7)$ & $0(0)$ & $1(8.3)$ & \\
\hline $\mathrm{SUV}_{\max -\mathrm{N}}>10.58$ & 8 & $3(37.5)$ & $2(25)$ & $3(37.5)$ & \\
\hline MTV & & & & & 0.018 \\
\hline$\leq 25.8 \mathrm{~cm}^{3}$ & 12 & $11(91.7)$ & $1(8.3)$ & $0(0)$ & \\
\hline$>25.8 \mathrm{~cm}^{3}$ & 8 & $3(37.5)$ & 1 (12.5) & $4(50)$ & \\
\hline Histology & & & & & 0.715 \\
\hline SCC - keratinising & 4 & $3(75)$ & $1(25)$ & $0(0)$ & \\
\hline Non-keratinising poorly differentiated & 7 & $4(57.1)$ & $1(14.3)$ & $2(28.6)$ & \\
\hline Non-keratinising undifferentiated & 9 & $7(77.8)$ & $0(0)$ & $2(22.2)$ & \\
\hline
\end{tabular}

Values are presented as number (\%), SUV - standard uptake value, MTV - metabolic tumour volume, CR - complete response, PR - partial response,

$P D$ - progressive disease

Table 4. Metabolic markers as a predictor of response

$\begin{array}{lcccc} & \text { Sensitivity } & \text { Specificity } & \begin{array}{c}\text { Positive } \\ \text { predictive } \\ \text { value }\end{array} & \begin{array}{c}\text { Negative } \\ \text { predictive } \\ \text { value }\end{array} \\ \text { SUV }_{\text {max-N }} & 83.3 \% & 71.4 \% & 55.6 \% & 91 \% \\ \text { MTV } & 83.3 \% & 78.6 \% & 62.5 \% & 91.7 \%\end{array}$

Values are presented as number (\%), SUV - standard uptake value, MTV - metabolic tumour volume

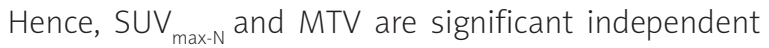
predictors of PFS and OS in univariate analysis, whereas it was not significant in the Cox regression multivariate analysis.
Table 5. Survival outcomes for nodal maximum standard uptake value and metabolic tumour volume

$\begin{array}{lccc}\begin{array}{l}\text { Prognostic } \\ \text { parameters }\end{array} & \text { SUV }_{\text {max-N }} \leq 10.58 & \text { SUV }_{\text {max-N }}>10.58 & p \text {-value } \\ \text { 2-year PFS } & 91 \% & 46 \% & 0.035 \\ \text { 2-year OS } & 95 \% & 58 \% & 0.015 \\ \text { Prognostic } & \text { MTV } \leq 25.8 & \text { MTV }>25.8 & p \text {-value } \\ \text { parameters } & & & \\ \text { 2-year PFS } & 90 \% & 38 \% & 0.006 \\ \text { 2-year OS } & 99 \% & 55 \% & 0.008\end{array}$

Values are presented as number (\%), SUV - standard uptake value, OS - overall survival, PFS - progression-free survival 

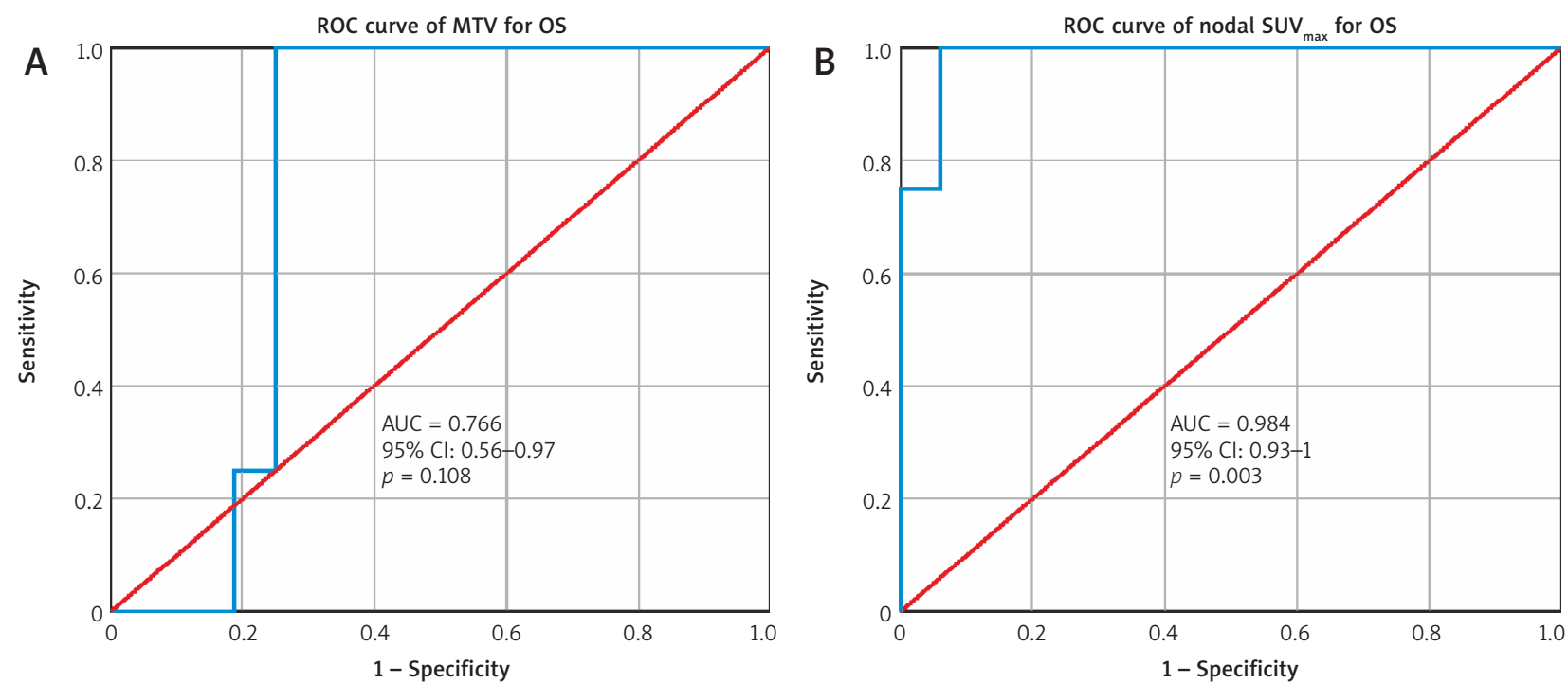

Fig. 2. Receiver operating characteristics curve for metabolic tumour volume and nodal maximum standard uptake value for predicting survival
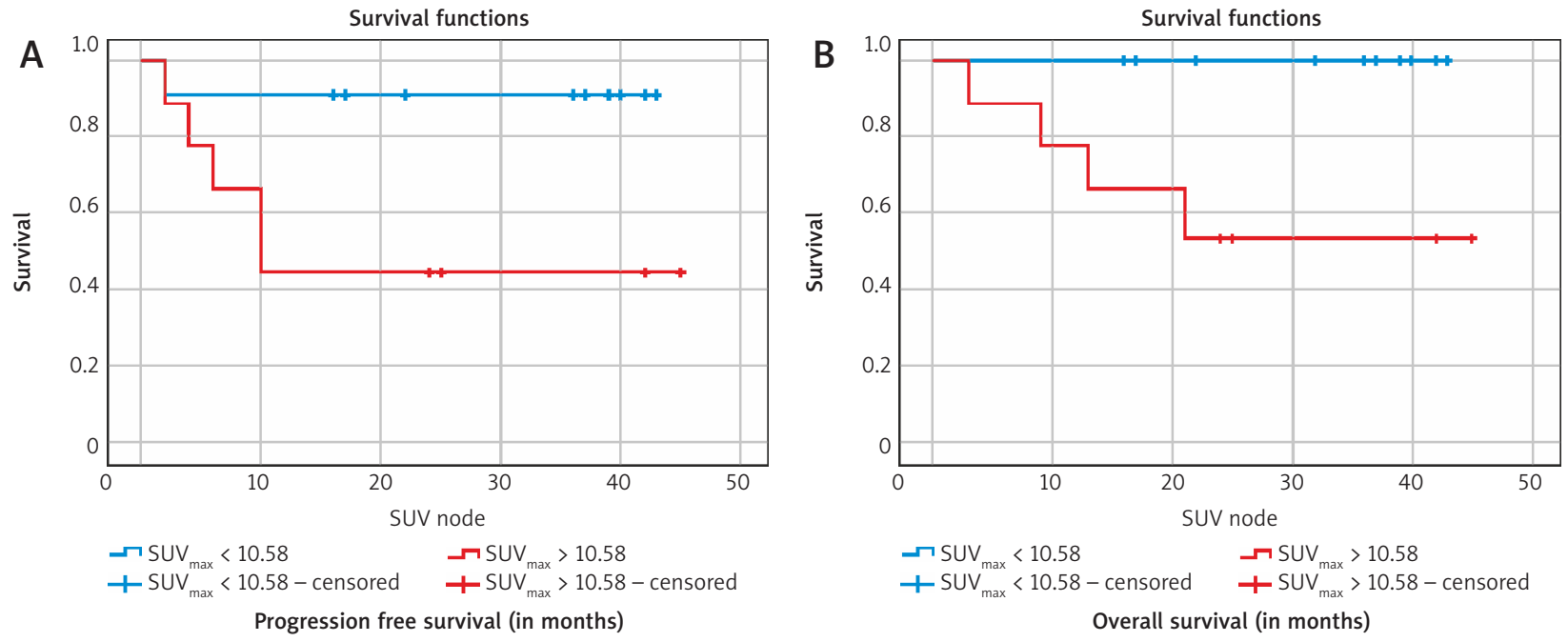

Fig. 3. A - Kaplan-Meier curves for progression-free survival, B - overall survival (nodal maximum standard uptake value)

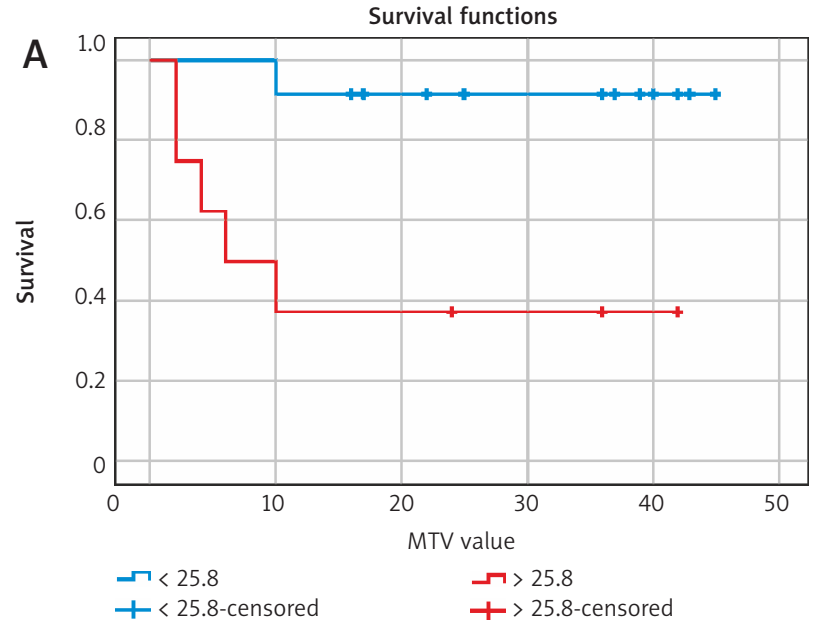

A. Progression free survival (in months)

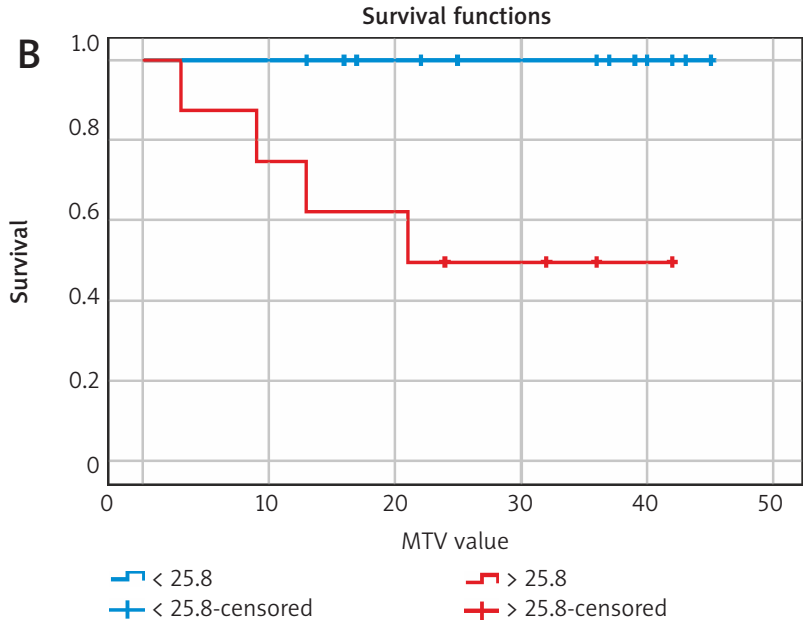

B. Overall survival (in months)

Fig. 4. A - Kaplan-Meier curves for progression-free survival, B - overall survival (metabolic tumour volume) 


\section{Discussion}

Although NPC is sensitive to chemoradiation, the OS rates for locally advanced cancers is around $71.3 \%$ with dismal distant failures rates. Hence, there is a need to identify factors that may predict prognostic outcomes, thereby leading to intensification of treatment for the poor prognostic subset. Among the prognostic factors in vogue are stage grouping, histological subgrouping, and assessment of tumour tissue microarrays [16, 17]. The main drawbacks of the previously mentioned factors are the lack of genetic expression of the entire tumour. Hence, identifying additional prognostic factors that can predict treatment outcomes, particularly non-invasive diagnostic methods, may enhance the development of individualised strategies, thereby improving treatment outcomes. PET-CT is a modality that is non-invasive and can obtain the entire tumour metabolic information.

${ }^{18}$ F-FDG-PET-CT is the gold standard imaging modality for staging and assessing treatment response in nasopharyngeal cancers. In addition to its use for staging workup, its metabolic parameters are being explored as prognostic markers that may dictate the treatment response as well as a marker to determine long-term survival outcomes. SUV is one such marker that has been evaluated in the reviewed literature. SUV is defined as the ratio of the radioactivity concentration measured in the tissue to body weight in kilograms. SUV ${ }_{\max }$ is a convenient parameter that is commonly used in clinical practice. It represents the highest voxel value within the volume of interest; however, it does not reveal heterogeneity within the tumour volume. Hence, other parameters, such as MTV, have been explored to offset this issue. In our study, we analysed the value of SUV $V_{\text {max-P' }}$ SUV $V_{\text {max-N }}$, and MTV as significant prognostic markers.

In terms of SUV $V_{\text {max-p. }}$, Xie et al. found the best cut-off value to be 8.0 for the SUV max $_{\text {max }}$ of primary although in multivariate analysis it was not statistically significant in terms of 5-year OS and 5-year PFS benefit [18]. Lee et al. also deemed a cut-off value of 8 to be optimum for prognosis prediction [19]. In contrast, in this study, the SUV $_{\text {max-p }}$ cut-off of 13.55 did not have any major bearing on the response rates or long-term survival outcomes, but

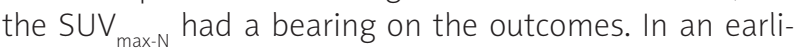
er retrospective study on locally advanced head and neck cancer patients from India Srinivas et al. [20] failed to identify a cut-off value for pretherapy SUV ${ }_{\max }$ that could predict the probable outcome of therapy.

In continuation of the above data obtained in this study in terms of SUV $V_{\max -\mathrm{N}}$ it should be noted that lymph node metastasis has long been an important independent prognostic factor for nasopharyngeal cancer patients. Various parameters for quantifying the prognostic value of lymph node status, such as MTV and SUV, have been studied by many investigators, although with conflicting data.

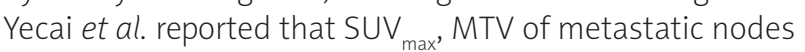
did not have a bearing as an independent prognostic factor [21]. However, in a study by Hung et al. SUV was deemed an independent prognostic factor, with distant metastasis-free survival of $79.9 \%$ [22, 23]. Similarly,
Lee et al. in their study also confirmed that the SUV max-N $_{\text {}}$ was a more sensitive marker with a higher pre-treatment value >13.4, which was a negative prognostic factor in terms of survival and disease progression (93.1\% vs. 55.5\% for OS and $92.7 \%$ vs. $38.5 \%$ for PFS) [24]. The data from this study are also in agreement with a PFS of $91 \%$ vs. $46 \%$ $(p=0.035)$ and an OS of 95\% vs. 58\% ( $p=0.015)$, which was statistically significant in our study.

Regarding MTV, the follow-up data of 20 patients in the present study showed that for a definite SUV threshold, the most distinct MTV cut-off value had prognostic merit. This suggests that the 2-year PFS rate was inversely related to the MTV cut-off value of $25.8 \mathrm{~cm}^{3}$ for an SUV threshold of 2.5, with the PFS being $91 \%$ vs. $46 \%$ ( $p=0.048$ ). Fei et al. also confirmed MTV as an independent prognostic factor for predicting treatment and survival outcomes in patients and deemed MTV at an SUV threshold of 4 as an ideal sub-volume to be chosen for best therapeutic effect [25]. Similarly, Chan et al. further highlighted the role of MTV as an independent risk factor in patients with metastatic NPC [14].

Thus, it may be reasonable to use MTV as a biological target volume for the sub-set of patients with higher MTV value. The functional information provided by the MTV and its application as a biological target volume and dose escalation volume in radiation planning may improve the therapeutic efficacy for patients with NPC, who receive concurrent chemoradiation. The threshold by which the MTV can be considered the biological target volume needs to be explored in future studies. The MTV, as measured by PET-CT, tends to be smaller than the gross tumour volume defined by MRI or CT $[26,27]$. Hence, the benefit regarding the use of dose escalation to a subregion within the GTV for a particular threshold of MTV and its effect on treatment or survival outcomes needs to be analysed exhaustively. This is beyond the scope of the present study and should be prospectively analysed in future studies.

A major limitation of our study was the retrospective nature and the limited sample size owing to the relatively low incidence of nasopharyngeal cancers in the southern districts of India. A well-designed prospective study is needed to validate the results of this study. Nevertheless, our report is still worthwhile because we have compared various metabolic parameters and conclusions were drawn with regard to MTV and SUV ${ }_{\text {max-N }}$, which has been shown to be a superior prognostic marker to SUV max.P. $_{\text {. }}$

\section{Conclusions}

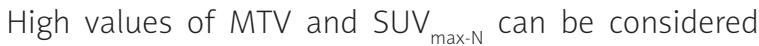
as independent prognostic factors of OS and PFS in nasopharyngeal cancer patients treated with concurrent chemoradiation, highlighting the need for more intense treatment for this subset.

The authors declare no conflict of interest. 


\section{References}

1. Ferlay J, Soerjomataram I, Dikshit R, et al. Cancer incidence and mortality worldwide: Sources, methods and major patterns in GLOBOCAN 2012. Int J Cancer 2015; 136: E359-86.

2. Stelow EB, Wenig BM. Update from the $4^{\text {th }}$ edition of the World Health Organization classification of head and neck tumours: nasopharynx. Head Neck Pathol 2017; 11: 16-22.

3. Lu H, Peng L, Yuan X, et al. Concurrent chemoradiotherapy in locally advanced nasopharyngeal carcinoma: a treatment paradigm also applicable to patients in Southeast Asia. Cancer Treat Rev 2009; 35: 345-353.

4. Zhao W, Lei H, Zhu X, Li L, Qu S, Liang X. Investigation of long-term survival outcomes and failure patterns of patients with nasopharyngeal carcinoma receiving intensity- modulated radiotherapy: a retrospective analysis. Oncotarget 2016; 7: 86914-86925.

5. Guo Q, Lu T, Lin S, et al. Long-term survival of nasopharyngeal carcinoma patients with Stage II in intensity-modulated radiation therapy era. Jpn J Clin Oncol 2016; 46: 241-247.

6. Aribas BK, Cetindag F, Ozdogan Z, et al. Nasopharyngeal carcinomas: prognostic factors and treatment features. J Egypt Natl Canc Inst 2008; 20: 230-236.

7. Feng M, Wang W, Fan Z, et al. Tumor volume is an independent prognostic indicator of local control in nasopharyngeal carcinoma patients treated with intensity-modulated radiotherapy. Radiat Oncol 2013; 8: 208

8. Ou X, Yang Z, Hu C. Use of ${ }^{18}$ F-FDG-PET-CT in the diagnosis, staging, response assessment and prognosis of nasopharyngeal carcinoma: an updated review. Clin Cancer Res 2014; 13: 2627-2633.

9. Higgins KA, Hoang JK, Roach MC, et al. Analysis of pretreatment FDG-PET SUV parameters in head-and-neck cancer: tumor SUV mean has superior prognostic value. Int I Radiat Oncol Biol Phys 2012; 82: 548-553.

10. Schwartz DL, Rajendran J, Yueh B, et al. FDG-PET prediction of head and neck squamous cell cancer outcomes. Arch Otolaryngol Head Neck Surg 2004; 130: 1361-1367.

11. Chang KP, Tsang NM, Liao CT, et al. Prognostic significance of ${ }^{18}$ F-FDG-PET parameters and plasma Epstein-Barr virus DNA load in patients with nasopharyngeal carcinoma. J Nucl Med 2012; 53: 21-28

12. Chan WK, Kwong DL, Yeung DW, et al. Prognostic impact of standardized uptake value of F-18 FDG PET/CT in nasopharyngeal carcinoma. Clin Nucl Med 2011; 36: 1007-1011.

13. Dibble EH, Alvarez ACL, Truong MT, et al. ${ }^{18} \mathrm{~F}-\mathrm{FDG}$ metabolic tumor volume and total glycolytic activity of oral cavity and oropharyngeal squamous cell cancer: adding value to clinical staging. J Nucl Med 2012; 53: 709-715.

14. Sheng-Chieh C, Cheng-Lung H, Tzu-Chen Y, et al. The role of ${ }^{18} \mathrm{~F}-\mathrm{FDG}$-PET-CT metabolic tumour volume in predicting survival in patients with metastatic nasopharyngeal carcinoma. Oral Oncol 2013; 49: 71-78

15. Lee AW, Ng WT, Pan JJ, et al. International guideline for the de lineation of the clinical target volumes (CTV) for nasopharyngeal carcinoma. Radiother Oncol 2018; 126: 25-36.

16. Nakao K, Mochiki M, Nibu K, Sugasawa M, Uozaki H. Analysis of prognostic factors of nasopharyngeal carcinoma: impact of in situ hybridization for Epstein-Barr virus encoded small RNA 1. Otolaryngol Head Neck Surg 2006; 134: 639-645.

17. Lee SW, Cho KJ, Park JH, et al. Expressions of Ku70 and DNAPKcs as prognostic indicators of local control in nasopharyngeal carcinoma. Int J Radiat Oncol Biol Phys 2005; 62: 1451-1457.

18. Xie P, Yue JB, Fu Z, Feng R, Yu JM. Prognostic value of ${ }^{18} \mathrm{~F}$-FDG-PETCT before and after radiotherapy for locally advanced nasopharyngeal carcinoma. Ann Oncol 2010; 21: 1078-1082.

19. Lee SW, Nam SY, Im KC, et al. Prediction of prognosis using standardized uptake value of 2-18 F-fluoro-2-deoxy-d-glucose positron emission tomography for nasopharyngeal carcinomas. Radiother Oncol 2008; 87: 211-216.

20. Srinivas KS, Arunan M, Venkatachalapathy E, John C, Manickavasagam M, Divyambika CV. The prognostic role of maximum standardized uptake value of ${ }^{18} \mathrm{~F}$-flourodeoxy glucose positron emission tomography-computed tomography in head and neck cancer patients undergoing chemoradiotherapy. J Int Soc Prev Community Dent 2019; 9: 159-165.

21. Huang Y, Feng $M$, He Q, et al. Prognostic value of pretreatment ${ }^{18} \mathrm{~F}-\mathrm{FDG}$-PET-CT for nasopharyngeal carcinoma patients. Medicine (Baltimore) 2017; 96: e6721.

22. Hung TM, Fan KH, Kang CJ, et al. Lymph node-to-primary tumor standardized uptake value ratio on PET predicts distant metastasis in nasopharyngeal carcinoma. Oral Oncol 2020; 110: 104756.

23. Hung TM, Wang HM, Kang CJ, et al. Pretreatment (18)F-FDG PET standardized uptake value of primary tumor and neck lymph nodes as a predictor of distant metastasis for patients with nasopharyngeal carcinoma. Oral Oncol 2013; 49: 169-174.

24. Lee SJ, Kay CS, Kim YS, et al. Prognostic value of nodal SUV of ${ }^{18}$ F-FDG-PET-CT in nasopharyngeal carcinoma treated with intensity-modulated radiotherapy. Radiat Oncol J 2017; 35: 306-316.

25. Fei Z, Chen C, Huang Y, et al. Metabolic tumor volume and conformal radiotherapy based on prognostic PET/CT for treatment of nasopharyngeal carcinoma. Medicine (Baltimore) 2019; 98: e16327.

26. Liu F, Xi XP, Wang H, et al. PET/CT-guided dose-painting versus CT-based intensity modulated radiation therapy in locoregional advanced nasopharyngeal carcinoma. Radiat Oncol 2017; 12: 15.

27. Wang J, Zheng J, Tang T, et al. A randomized pilot trial comparing position emission tomography (PET)-guided dose escalation radiotherapy to conventional radiotherapy in chemoradiotherapy treatment of locally advanced nasopharyngeal carcinoma. PLoS One 2015; 10: e0124018.

\section{Address for correspondence}

\section{Christopher John}

Department of Radiation Oncology

Sri Ramachandra Institute

of Higher Education and Research

Porur, Chennai-600116, India

e-mail: chriscj1808@gmail.com

Submitted: 02.08 .2021

Accepted: 19.08.2021 